\title{
Sweep imaging with Fourier transform as a tool with MRI for evaluating the effect of teriparatide on cortical bone formation in an ovariectomized rat model
}

Yasutaka Sotozono ${ }^{1}$, Kazuya Ikoma ${ }^{1 *}$, Masamitsu Kido ${ }^{1}$, Okihiro Onishi ${ }^{1}$, Masataka Minami ${ }^{1}$, Hiroaki Wada', Shunji Yamada ${ }^{2}$, Ken-Ichi Matsuda ${ }^{2}$, Masaki Tanaka² and Kenji Takahashi ${ }^{1}$

\begin{abstract}
Background: Teriparatide (TPTD) is a drug for osteoporosis that promotes bone formation and improves bone quality. However, the effects of TPTD on cortical bone are not well understood. Sweep imaging with Fourier transform (SWIFT) has been reported as a useful tool for evaluating bound water of cortical bone, but it has yet to be used to investigate the effects of TPTD on cortical bone. This study aimed to evaluate the consequences of the effect of TPTD on cortical bone formation using SWIFT.
\end{abstract}

Methods: Twelve-week-old female Sprague-Dawley rats $(n=36)$ were reared after ovariectomy to create a postmenopausal osteoporosis model. They were divided into two groups: the TPTD and non-TPTD groups. Rats were euthanized at 4, 12, and 24 weeks after initiating TPTD treatment. Tibial bones were evaluated using magnetic resonance imaging (MRI) and bone histomorphometry. In MRI, proton density-weighted imaging (PDWI) and SWIFT imaging were performed. The signal-to-noise ratio (SNR) was calculated for each method. The same area evaluated by MRI was then used to calculate the bone formation rate by bone histomorphometry. Measurements were compared using the Mann-Whitney U-test, and a P-value of $<0.05$ was considered significant.

Results: PDWI-SNR was not significantly different between the two groups at any time point $(P=0.589,0.394$, and 0.394 at 4, 12, and 24 weeks, respectively). Contrarily, SWIFT-SNR was significantly higher in the TPTD group than in the non-TPTD group at 4 weeks after initiating treatment, but it was not significantly different at 12 and 24 weeks ( $P=0.009,0.937$, and 0.818 at 4,12 , and 24 weeks, respectively). The bone formation rate assessed by histomorphometry was significantly higher in the TPTD group than in the non-TPTD group at all timepoints $(P<0.05$, all weeks). In particular, at 4 weeks, the bone formation rate was markedly higher in the TPTD group than in the non-TPTD group $\left(P=0.028,1.98 \pm 0.33\right.$ vs. $0.09 \pm 0.05 \mu^{3} / \mu \mathrm{m}^{2} /$ day $)$.

Conclusions: SWIFT could detect increased signals of bound water, reflecting the effect of TPTD on the cortical bone. The signal detected by SWIFT reflects a marked increase in the cortical bone formation rate.

Keywords: Osteoporosis, Teriparatide, Sweep imaging with Fourier transform

\footnotetext{
*Correspondence: kazuya@koto.kpu-m.ac.jp

${ }^{1}$ Department of Orthopaedics, Graduate School of Medical

Science, Kyoto Prefectural University of Medicine, 465 Kajii-cho, Kawaramachi-Hirokoji, Kamigyo-ku, Kyoto, Japan

Full list of author information is available at the end of the article
}

\section{Background}

Osteoporosis is defined as "a skeletal disorder characterized by compromised bone strength predisposing to an increased risk of fracture" [1]. Bone strength is defined original author(s) and the source, provide a link to the Creative Commons licence, and indicate if changes were made. The images or other third party material in this article are included in the article's Creative Commons licence, unless indicated otherwise in a credit line to the material. If material is not included in the article's Creative Commons licence and your intended use is not permitted by statutory regulation or exceeds the permitted use, you will need to obtain permission directly from the copyright holder. To view a copy of this licence, visit http://creativecommons.org/licenses/by/4.0/. The Creative Commons Public Domain Dedication waiver (http://creativeco mmons.org/publicdomain/zero/1.0/) applies to the data made available in this article, unless otherwise stated in a credit line to the data. 
by bone mineral density (BMD) and bone quality, both of which are closely related to bone turnover $[1,2]$. Postmenopausal osteoporosis is the most common type of osteoporosis [3], and an increase of bone turnover, bone resorption exceeding bone formation, is associated with decreased BMD and bone quality, as well as an increased risk of fracture [4]. Therefore, it is necessary to improve bone turnover to treat postmenopausal osteoporosis, and to this end, various therapeutic drugs have been developed. Teriparatide (TPTD) is a drug for the treatment of osteoporosis that promotes bone formation with intermittent administration [5]. Various studies have been conducted on the promotion of osteogenesis by TPTD [5-7]. However, the site and time at which osteogenesis occurs in the bone remain unclear.

The bone structure is composed of cortical and cancellous bones. Of the two types, the cortical bone comprises the majority, helps support body weight, and prevents fractures $[8,9]$. These findings emphasize the significance of evaluating the cortical bone. However, dual-energy X-ray absorptiometry (DXA), which is commonly used for the diagnosis of osteoporosis, cannot be restricted to the cortical bone only.

Sweep imaging with Fourier transform (SWIFT) is a novel imaging method in magnetic resonance imaging (MRI) that can depict proton signals of bound water with very short $T_{2}$ relaxation times that cannot be measured by conventional MRI [10]. It has been reported that the signals of bound water detected using SWIFT was associated with bone formation in the cortical bone of postmenopausal osteoporotic and diabetic osteoporotic rats $[11,12]$. The signal-to-noise ratio (SNR) performance is better with SWIFT than with conventional imaging, although $\mathrm{T}_{2}$ sensitivity is the same [13]. Therefore, it is reasonable to expect that the effect of TPTD on bone formation in the cortical bone could be evaluated using SWIFT in a site-specific and time-specific manner.

This study aimed to evaluate the consequences of the effect of TPTD on cortical bone formation using SWIFT. Furthermore, we verified whether SWIFT could be used to determine the therapeutic effect of TPTD in osteoporosis.

\section{Methods}

\section{Animal model}

Twelve-week-old female Sprague-Dawley rats $(n=36$; Shimizu Laboratory Supplies, Kyoto, Japan) were used in this study. The rats were kept at the animal facilities of our institute according to the Guide for the Care and Use of Laboratory Animals published by the National Institutes of Health. This study was approved by the Institutional Review Board for animal experiments and carried out in compliance with ARRIVE guidelines. Four rats were housed per transparent plastic cage. The temperature was maintained at $23-24^{\circ} \mathrm{C}$ at our institution, and light-dark cycles were performed every $12 \mathrm{~h}$. All animals were able to consume food and water ad libitum. All rats underwent ovariectomy under aseptic conditions, and $1.5 \%$ isoflurane was used for anesthesia. They were reared for 12 weeks after ovariectomy to create a postmenopausal osteoporosis model [14]. The rats were then randomly divided into the following two groups: TPTD and nonTPTD. For the TPTD group, TPTD was administered at $30 \mu \mathrm{g} / \mathrm{kg} /$ day, three times per week starting 12 weeks after the ovariectomy [6]. The non-TPTD group received normal saline at the same volume. The rats were euthanized by the intravenous injection of $4.0 \mathrm{~mL}$ of pentobarbital sodium (Somnopentyl ${ }^{\circledR}$, Kyoritsuseiyaku Corp., Tokyo, Japan) at 4, 12, and 24weeks following the initiation of TPTD treatment. Various examinations were performed according to the protocol described below. Figure 1 shows the regions of interest (ROIs) in the various tests.

\section{MRI}

The tibia on the right side of the euthanized rats was removed and evaluated using MRI. We used a high-field MRI machine (Varian MRI system 7.04 T, Agilent Technologies, Palo Alto, CA) and a transmit-and-receive surface coil $(3 \times 3 \mathrm{~mm}$ in diameter $)$ for animal experimentation purposes. We confirmed that the measurement errors of the MRI machine were less than $1 \%$. A container with a sample placed at the center of the surface coil was installed. The specimens were all placed with the same orientation to ensure an identical flip angle (FA). SWIFT was performed using the following protocol: field of view (FOV) of $40 \times 240 \times 40 \mathrm{~mm}^{3}$; matrix of $256 \times 256 \times 256 ; 16$ spirals; 8192 views; resolution of $0.156 \times 0.938 \times 0.156 \mathrm{~mm}^{3}$; bandwidth of $62.5 \mathrm{kHz}$; repetition time (TR) of $12.5 \mathrm{~ms}$; FA of $25^{\circ}$; acquisition time of $4.096 \mathrm{~ms}$; and scan time of $27 \mathrm{~min} 25 \mathrm{~s}$. The proton density-weighted image (PDWI) was obtained according to the following protocol using a conventional fast spin echo method: FOV of $40 \times 40 \mathrm{~mm}^{2}$; matrix of $256 \times 256$; resolution of $0.156 \times 0.156 \mathrm{~mm}^{2}$; axial slice of 30; slice thickness of $1 \mathrm{~mm}$; bandwidth of $100 \mathrm{kHz}$; TR of $2000 \mathrm{~ms}$; echo spacing of $9.54 \mathrm{~ms}$; echo train length of 4 ; acquisition time of $2.56 \mathrm{~ms}$; and scan time of $2 \mathrm{~min} 13 \mathrm{~s}$. These protocols were similar to those described in previous reports $[10,11]$. The images were analyzed using a software program (ImageJ v. 1.49, National Institutes of Health, Bethesda, MD). The ROI was the entire cortical bone region of the tibia in a transverse section, which was $200 \mu \mathrm{m}$ in thickness and $2 \mathrm{~mm}$ proximal to the tibiofibular union. The quality of signals in the SWIFT and PDWI were measured and averaged. The SNR was defined as the mean value of the quality of signals in the 


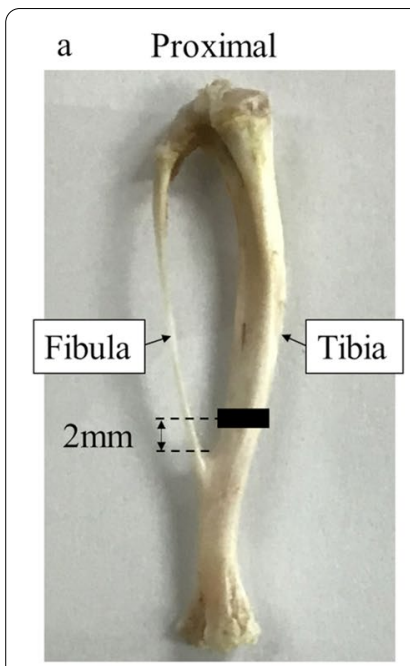

Distal

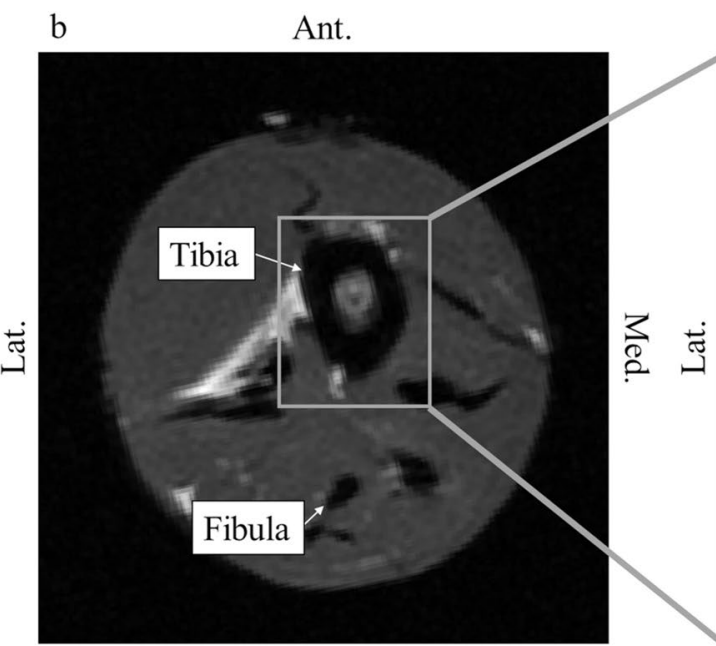

Post.

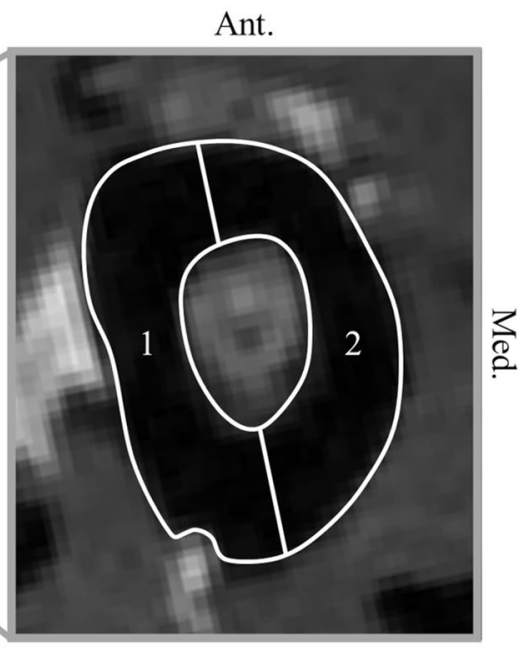

Post.

Fig. 1 Regions of interest for MRI. a The regions are set $2 \mathrm{~mm}$ proximal to the tibiofibular union. $\mathbf{b}$ Two regions, including all the areas of cortical bone, are set in the cortical bone of the tibia in the axial plane (proton density-weighted image). Ant. = anterior, Post. = posterior, Med. = medial, Lat. $=$ lateral

$\mathrm{ROI} /$ standard deviation of the quality of signals in the background, SWIFT-SNR, and PDWI-SNR, respectively.

\section{DXA}

The left tibia was removed from euthanized rats and evaluated using DXA (DCS-600EX-R; Aloka, Tokyo, Japan). We confirmed that the measurement errors of the DXA were less than $2 \%$. The ROI was the entire tibial region in an area, $200 \mu \mathrm{m}$ in thickness, and $2 \mathrm{~mm}$ proximal to the tibiofibular union. BMD in the ROI was measured.

\section{Microcomputed tomography $(\mu \mathrm{CT})$}

DXA was followed by $\mu C T$ (micro-focus 2D/3D, ScanXmate-E090S40, Comscantecno, Kanagawa, Japan) of the tibia on the left side with the following settings: voltage of $60 \mathrm{kV}$, electric current of $85 \mu \mathrm{A}$, and voxel size of $37.5 \mu \mathrm{m}$. We confirmed that the measurement errors of the $\mu \mathrm{CT}$ were less than $1 \%$. Three-dimensional reconstructed images were created using image reconstruction software (ConeCTexpress v. 1.3, Comscantecno, Kanagawa, Japan). The ROI was the entire cortical bone region of the tibia, which was the same site used for the MRI. The volumetric BMD (vBMD) of the cortical bone and the cortical bone width were measured using image analysis software (TRI/3D-BON, Ratoc System Engineering, Tokyo, Japan).

\section{Bone histomorphometry}

Among the tibias on the right side that underwent MRI, four from each group also underwent bone histomorphometry each week. Approximately $5 \mathrm{mg}$ of calcein (SigmaAldrich) was injected subcutaneously into the back of the tibia at 4 and 10 days prior to removal to indicate areas of the new bone. The dosing interval for calcein was $144 \mathrm{~h}$ (6 days). The tibia was fixed with $70 \%$ alcohol immediately after the MRI. Specimens were stained with Villanueva bone stain to assess the sites identical to those measured by MRI. The bone surface (BS, $\mu \mathrm{m})$, porosity area (Po Ar), and cortical bone area ( $\mathrm{Ct} \mathrm{Ar}$ ) were measured using Parfitt's method [15]. The Haversian canal and Volkmann's canal were included in the bone pores, and the osteocyte gap was excluded from the bone pore. Po $\mathrm{Ar} / \mathrm{Ct} \mathrm{Ar}$ (\%) was measured in the cortical bone areas. $\mathrm{BS}$ and $\mathrm{Ct}$ Ar were measured under natural light at $\times 100$ magnification and Po Ar was measured at $\times 200$ magnification.

Under Fluorinert fluorescence, the single-labeled surface (sLs, $\mu \mathrm{m})$, double-labeled surface (dLs, $\mu \mathrm{m})$, and mineral apposition rate (MAR, $\mu \mathrm{m} /$ day) were measured using calcein labeling as an index. Both sLs and dLs were measured in the endosteum and periosteum. MAR was measured as the width of labeling produced by the injection at 144-h intervals in 10 selected sites at the most obvious sites of bone formation. The bone formation rate (BFR/BS, $\mu \mathrm{m}^{3} / \mu \mathrm{m}^{2} /$ day) was measured as BFR/ $\mathrm{BS}=(\mathrm{sLs}+2 \mathrm{dLs}) \times \mathrm{MAR} / \mathrm{BS}$. Measurements under Fluorinert fluorescence were performed at a magnification of $\times 200$.

\section{Statistical analysis}

Results are presented as the mean \pm standard deviation. Results of DXA, $\mu \mathrm{CT}$, MRI, and bone histomorphometry measured at each week in the TPTD and non-TPTD 
groups were compared using the Mann-Whitney U-test. Differences were considered significant at a $P$-value of $<0.05$. EZR (Saitama Medical Center, Jichi Medical University, Saitama, Japan), a statistical software program that extends the functions of $\mathrm{R}$ and $\mathrm{R}$ Commanders [16], was used for all statistical analyses.

\section{Results}

MRI

Figure 2 shows the SNR within the cortical bone measured by MRI. The PDWI-SNRs were $4.6 \pm 0.8,4.3 \pm 0.4$, and $3.6 \pm 0.5$ in the TPTD group, and $5.1 \pm 1.3,4.0 \pm 0.7$, and $4.1 \pm 0.7$ in the non-TPTD group at 4,12 , and 24 weeks, respectively, after the initiation of TPTD treatment. There was no significant difference between the TPTD and non-TPTD groups at any time point ( $P=0.589,0.394,0.394$ at 4,12 , and 24 weeks, respectively). On the other hand, the SWIFT-SNRs were $12.6 \pm 3.5,5.4 \pm 0.9$, and $5.6 \pm 0.6$ in the TPTD group and $7.6 \pm 1.6,5.5 \pm 1.4$, and $5.6 \pm 0.9$ in the non-TPTD group at 4,12 , and 24 weeks, respectively. A significant difference was observed at 4 weeks after the initiation of TPTD treatment, but no significant difference was observed at 12 or 24 weeks $(P=0.009,0.937$, and 0.818 at 4,12 , and
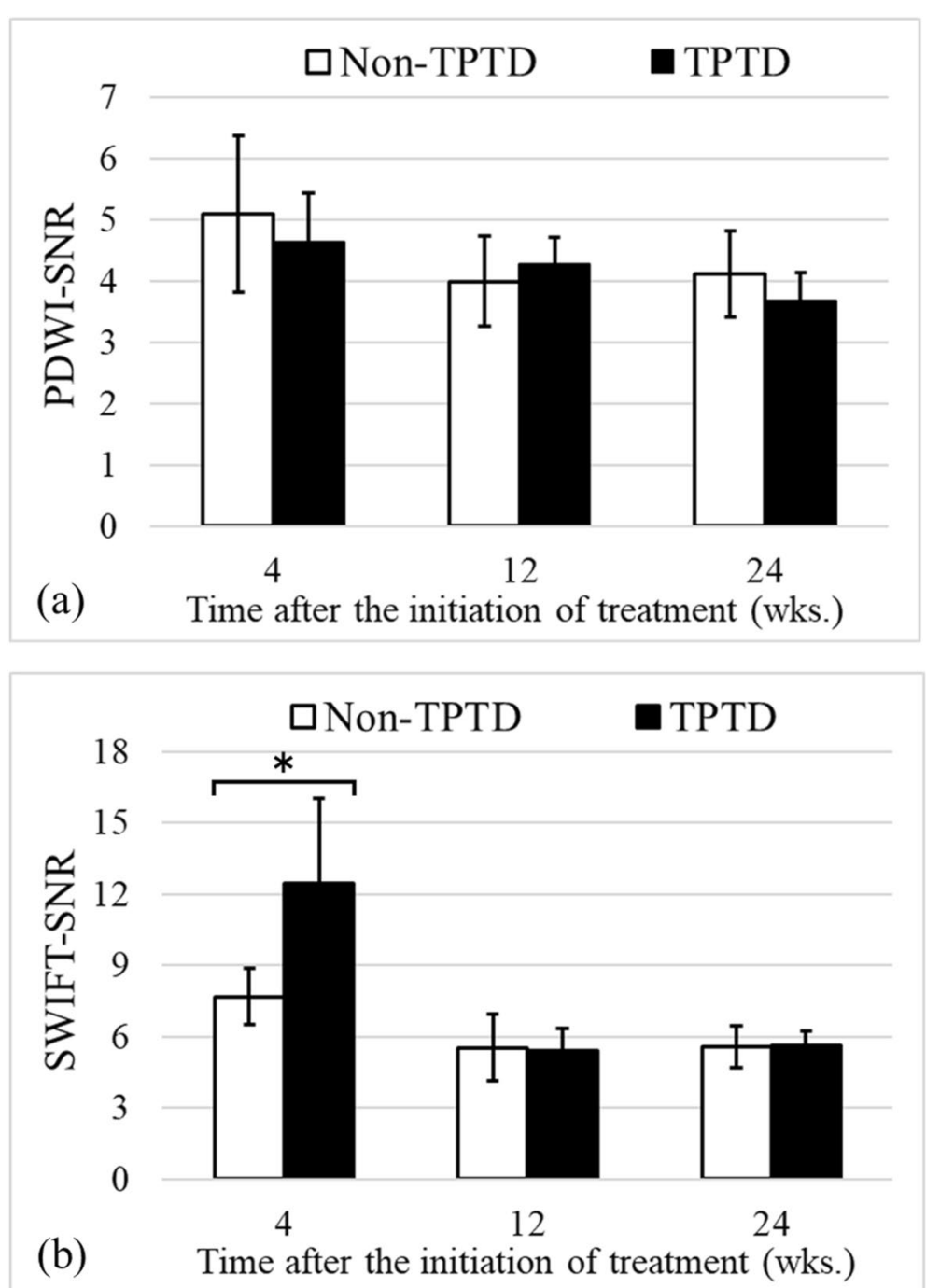

Fig. 2 The signal-to-noise ratio (SNR) within the tibial cortical bone measured by (a) proton density-weighted imaging (PDWI) and (b) sweep imaging with Fourier transform (SWIFT) for the teriparatide (TPTD) and non-TPTD groups. Values are expressed as means \pm standard deviations. There was no significant difference in the PDWI-SNR between the two groups at any time point $(P=0.589,0.394$, and 0.394 at 4, 12 , and 24 weeks, respectively). The SWIFT-SNR was significantly higher in the TPTD group than in the non-TPTD group at 4 weeks after the initiation of treatment, but no significant difference was observed at 12 and 24 weeks $(P=0.009,0.937$, and 0.818 at 4,12 , and 24 weeks, respectively). *: $P<0.05$ 
24 weeks, respectively). Figure 3 shows the results of PDWI and SWIFT representatives for both groups at 4, 12 , and 24 weeks after the initiation of TPTD treatment.

\section{DXA}

The BMD in all tibial regions measured by DXA at 4,12 , and 24 weeks was $99.9 \pm 4.0,104.4 \pm 5.0$, and $95.3 \pm 5.2 \mathrm{mg} / \mathrm{cm}^{2}$, respectively, in the TPTD group, and $89.7 \pm 4.3,98.5 \pm 3.0$, and $85.3 \pm 2.1 \mathrm{mg} / \mathrm{cm}^{2}$, respectively, in the non-TPTD group. The levels were significantly higher in the TPTD group than in the non-TPTD group at all timepoints $(P<0.05$ at all weeks $)$.

\section{$\mu C T$}

Figure 4 shows representative images obtained by $\mu C T$. The vBMDs of the cortical bone at 4,12 , and 24 weeks after the initiation of TPTD treatment were $1229 \pm 71,1231 \pm 93$, and $1236 \pm 84 \mathrm{mg} / \mathrm{cm}^{3}$ in the TPTD group, respectively, and $1190 \pm 112,1237 \pm 49$, and $1223 \pm 67 \mathrm{mg} / \mathrm{cm}^{3}$ in the non-TPTD group, respectively. There was no significant difference between the TPTD and non-TPTD groups at any time point $(P=0.699$, $0.937,0.818$ at 4,12 , and 24 weeks, respectively). The cortical bone width was $760 \pm 15,786 \pm 15$, and $802 \pm 26 \mu \mathrm{m}$ in the TPTD group and $709 \pm 16,723 \pm 13$, and $715 \pm 21 \mu \mathrm{m}$ in the non-TPTD group at 4,12 , and
24 weeks, respectively, after the initiation of TPTD treatment. The levels were significantly higher in the TPTD group than in the non-TPTD group at all timepoints $(P<0.05$ at all weeks). Table 1 shows the results of BMD measured by DXA, vBMD of cortical bone, and cortical bone width measured by $\mu \mathrm{CT}$.

\section{Bone histomorphometry}

Figure 5 shows Po Ar/Ct Ar and BFR/BS measured by bone histomorphometry. Po Ar/Ct Ar was $0.69 \pm 0.14$, $0.68 \pm 0.11$, and $0.16 \pm 0.08$ in the TPTD group, and $0.69 \pm 0.04,0.57 \pm 0.15$, and $0.30 \pm 0.06$ in the non-TPTD group at 4,12 , and 24 weeks, respectively, after the initiation of TPTD treatment. There was no significant difference between the TPTD and non-TPTD groups at any time point $(P=0.857,0.486$, and 0.057 at 4,12 , and 24weeks, respectively). BFR/BS was $1.98 \pm 0.33$, $0.34 \pm 0.19$, and $0.32 \pm 0.08 \mu \mathrm{m}^{3} / \mu^{2} /$ day in the TPTD group, and $0.09 \pm 0.06,0.02 \pm 0.02$, and $0.03 \pm 0.01 \mu \mathrm{m}^{3} /$ $\mu \mathrm{m}^{2} /$ day in the non-TPTD group at 4,12 , and 24 weeks, respectively, after the initiation of TPTD treatment. The values were significantly higher in the TPTD group than in the non-TPTD group at all timepoints $(P<0.05$ at all weeks). In particular, at 4 weeks, BFR/BS was markedly higher in the TPTD group than in the non-TPTD group.

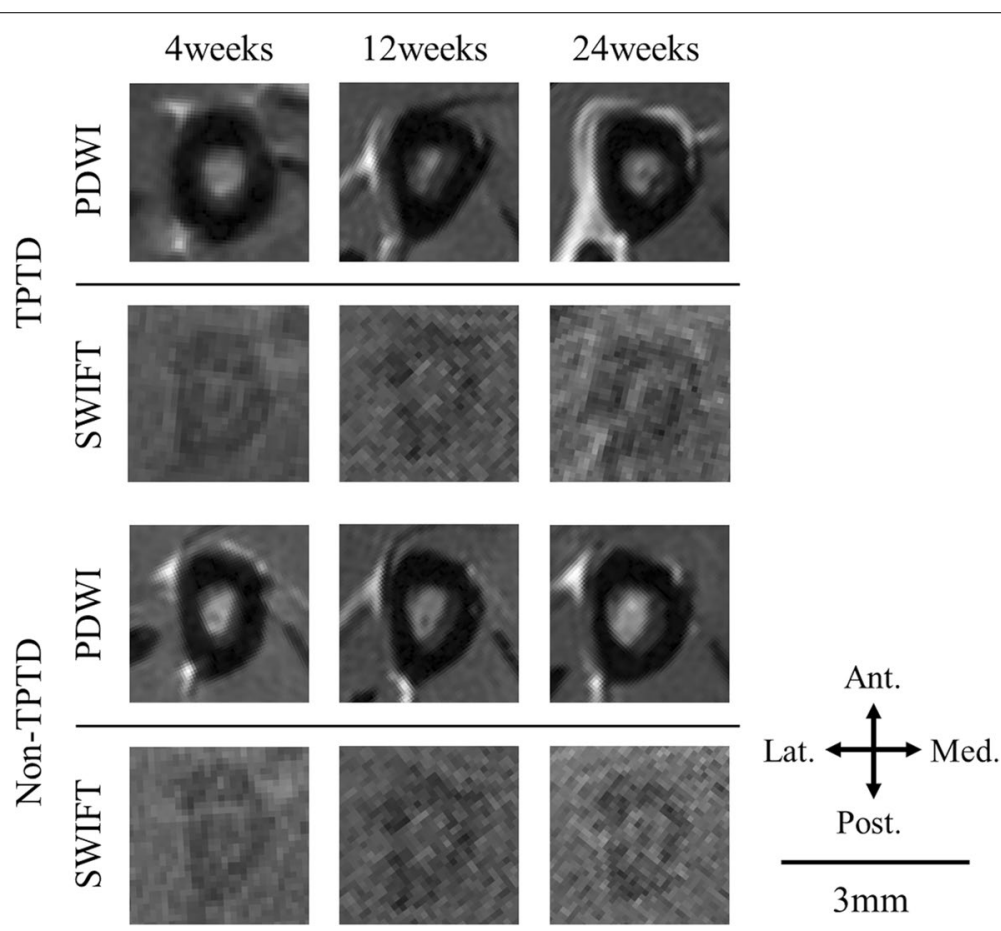

Fig. 3 Representative images obtained via proton density-weighted imaging (PDWI) and sweep imaging with Fourier transform (SWIFT) for the teriparatide (TPTD) and non-TPTD groups. SWIFT can detect proton signals in the cortical bone, which PDWI is unable to detect. Ant. = anterior, Post. $=$ posterior, Med. $=$ medial, Lat. $=$ lateral 


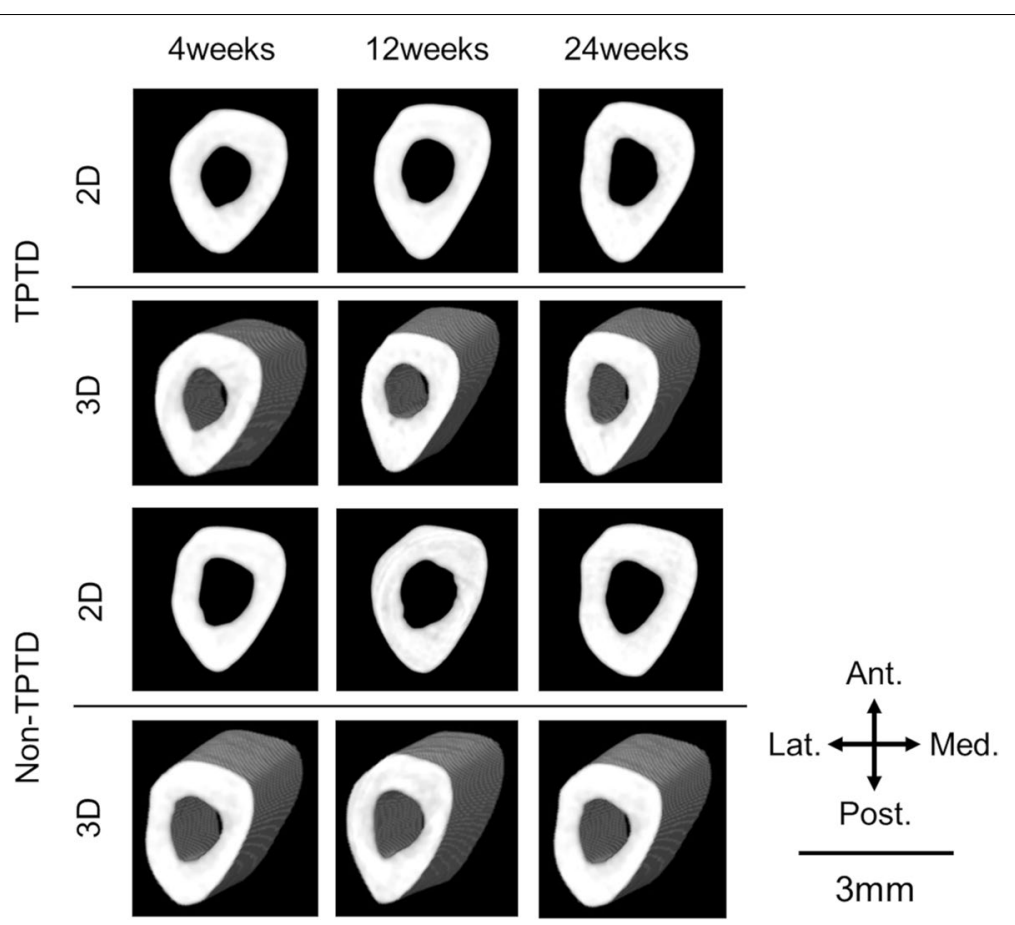

Fig. 4 Representative 2D and 3D images obtained via microcomputed tomography $(\mu C T)$ for the teriparatide (TPTD) and non-TPTD groups. Ant. $=$ anterior, Post. $=$ posterior, Med. $=$ medial, Lat. $=$ lateral

Table 1 Results of BMD measured by DXA, vBMD of cortical bone, and cortical width measured by $\mu C T$

\begin{tabular}{|c|c|c|c|c|}
\hline & $\begin{array}{l}\text { Time after the initiation of } \\
\text { treatment }\end{array}$ & Non-TPTD & TPTD & $P$-value \\
\hline \multicolumn{5}{|l|}{ DXA } \\
\hline \multirow[t]{3}{*}{$\mathrm{BMD}\left(\mathrm{mg} / \mathrm{cm}^{2}\right)$} & 4 weeks & $89.7 \pm 4.3$ & $99.9 \pm 4.0$ & $<0.01$ \\
\hline & 12 weeks & $98.1 \pm 3.0$ & $104.4 \pm 5.0$ & $<0.01$ \\
\hline & 24 weeks & $85.3 \pm 2.1$ & $95.3 \pm 5.2$ & $<0.05$ \\
\hline \multicolumn{5}{|l|}{$\mu C T$} \\
\hline \multirow[t]{3}{*}{ VBMD of cortical bone $\left(\mathrm{mg} / \mathrm{cm}^{3}\right)$} & 4 weeks & $1190 \pm 112$ & $1229 \pm 71$ & 0.67 \\
\hline & 12 weeks & $1237 \pm 49$ & $1231 \pm 93$ & 0.94 \\
\hline & 24 weeks & $1223 \pm 67$ & $1235 \pm 84$ & 0.82 \\
\hline \multirow[t]{3}{*}{ Cortical width $(\mu \mathrm{m})$} & 4 weeks & $709 \pm 16$ & $760 \pm 15$ & $<0.01$ \\
\hline & 12 weeks & $723 \pm 13$ & $786 \pm 15$ & $<0.01$ \\
\hline & 24 weeks & $714 \pm 22$ & $801 \pm 26$ & $<0.01$ \\
\hline
\end{tabular}

Values are expressed as means \pm standard deviations. BMD Bone mineral density, DXA Dual-energy X-ray absorptiometry, $v B M D$ Volumetric bone mineral density, $\mu C T$ Microcomputed tomography, TPTD Teriparatide

Figure 6 shows representative images of the bone formation rate.

\section{Discussion}

Our results suggest that it was possible to detect signals representing the effect of TPTD on cortical bone formation using SWIFT. The SWIFT-SNR was markedly increased early after the initiation of TPTD treatment, similar to the change in the bone formation rate in bone histomorphometry. We believe that TPTD promotes bone formation in the cortical bone to a greater extent in the early stage after the initiation of TPTD treatment, and SWIFT detects the signals emitted by the newly formed bone area. This change could not be detected using PDWI, a conventional MRI technique. The effects of TPTD on the cortical bone could be detected using 

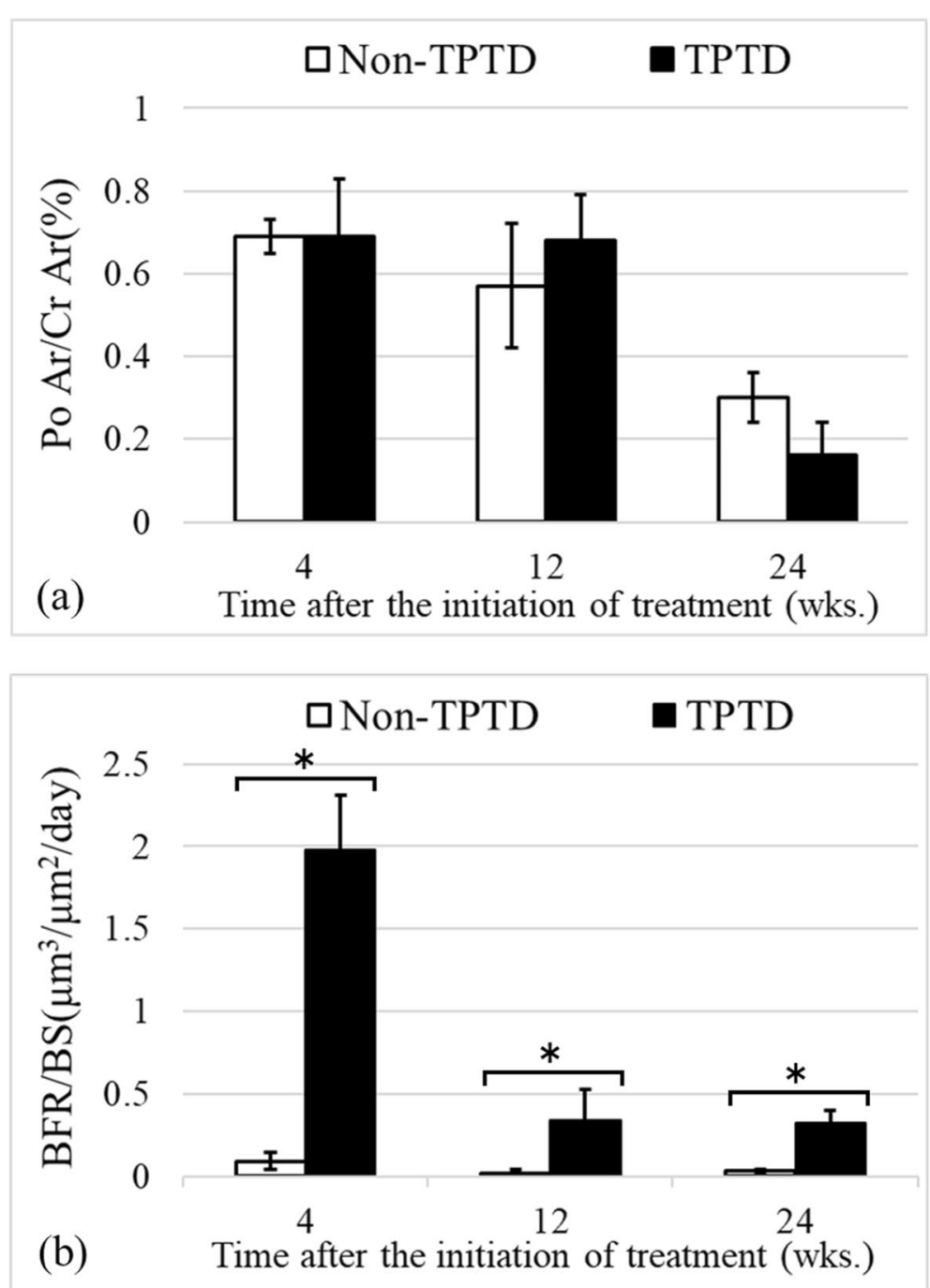

Fig. 5 a Porosity area/cortical area (Po Ar/Ct Ar) and $\mathbf{b}$ bone formation rate (BFR/BS) in the tibial cortical bone measured by bone histomorphometry. There was no significant difference in $\mathrm{Po} \mathrm{Ar} / \mathrm{Ct}$ Ar between the teriparatide (TPTD) and non-TPTD groups at any time point $(P=0.857,0.486$, and 0.0571 at 4, 12 , and 24 weeks, respectively). BFR/BS was significantly higher in the TPTD group than in the non-TPTD group at all timepoints $(P<0.05$ at all weeks). In particular, at 4 weeks, BFR/BS was markedly higher in the TPTD group than in the non-TPTD group. *: $P<0.05$

DXA and $\mu \mathrm{CT}$; however, a marked increase of bone formation early after the initiation of TPTD treatment could not be determined. The use of SWIFT enables the evaluation of the effect of TPTD on signals of bound water in a site- and time-specific manner.

DXA, which measures BMD, is the most commonly performed test for the treatment of osteoporosis [17]. The measurement results in this study were higher in the TPTD group than in the non-TPTD group, as in previous reports [18], confirming the appropriate administration of TPTD. DXA is a two-dimensional assessment method, and the measured BMD combines the cortical and cancellous bones. Therefore, it is not possible to only assess the cortical bone with DXA, even though the cortical bone has an important role in bone strength.

$\mu \mathrm{CT}$ is a three-dimensional assessment method that can be used to evaluate the cortical and cancellous bones separately [19]. Although the results of this study showed that the vBMD of the cortical bone did not differ at all weeks, the cortical bone width was higher in the TPTD group at all weeks, similar to previous reports [20]. TPTD may accelerate the cortical bone formation and increase the cortical bone width. $\mu \mathrm{CT}$ can be used to focus on the cortical bone. However, $\mu \mathrm{CT}$ is a mineral-based histomorphological assessment method and is therefore unable to assess non-mineral items. 


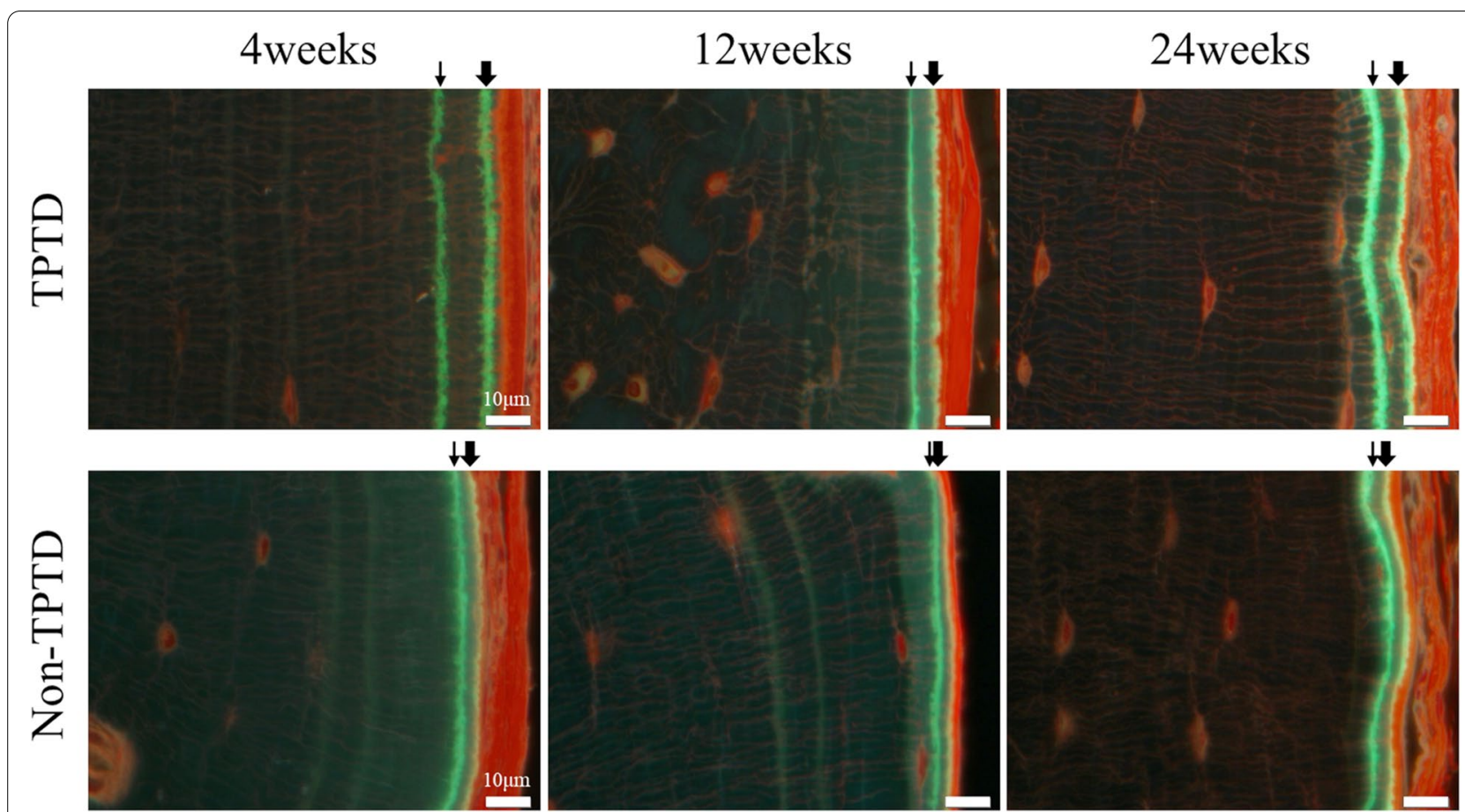

Fig. 6 Representative images of the areas of the new bone in the endosteum when observed under fluorescent light. The thin arrow indicates calcein injected at 10 days before tibial removal, and the thick arrow indicates calcein injected at 4 days before tibial removal. A marked increase in the bone formation rate in the teriparatide (TPTD) group was observed at 4 weeks after the initiation of TPTD treatment

Similar to $\mu \mathrm{CT}$, MRI is another three-dimensional assessment method. However, while $\mu \mathrm{CT}$ is used to assess minerals, MRI may be used to assess proton signals. The proton signal obtained from the cortical bone was from free and bound water [21]. The proton signal from free water has a millisecond $\mathrm{T}_{2}$ relaxation time and can be detected using PDWI, a conventional MRI method. On the other hand, the proton signal from bound water had a $T_{2}$ relaxation time of microseconds and could not be detected by PDWI [22]. In contrast, SWIFT can be used to detect signals with very short $\mathrm{T}_{2}$ relaxation times, allowing the detection of signals of bound water [10]. In this study, although the PDWI-SNR showed no differences at all weeks, the SWIFT-SNR was higher in the TPTD group than in the non-TPTD group only at 4 weeks after the initiation of TPTD treatment. It was possible to detect the effect of TPTD as a signal of an increase in the amount of bound water in the cortical bone early after the initiation of TPTD treatment.

TPTD has been reported to greatly improve bone formation in the early phase after treatment initiation $[7,23]$. With SWIFT, high signals were also detected at 4 weeks after the initiation of TPTD treatment, and the formation rate of the cortical bone in bone histomorphometry was also high at 4 weeks after the initiation of TPTD treatment compared with other weeks.
We believe that the signal detected by SWIFT reflects a marked increase in the bone formation rate in the cortical bone. Considering that bound water is abundant in the collagen in new bone areas and decreases with mineralization [24], the increase in the bone formation rate should be captured in SWIFT by detecting the signal of the bound water that exists in the collagen of new bone areas. From the present results, SWIFT seems to be more suitable to detect initial cortical bone formation than other imaging methods, such as PDWI, CT, and DXA. However, the increase in bone formation rate in the TPTD group at 12 and 24 weeks after the initiation of TPTD treatment could not be evaluated by SWIFT, possibly because the slight increase in the bone formation rate may have been insufficient to detect a signal difference.

A previous study has reported that the effect of TPTD on cortical bone porosity depends on the frequency of administration, increasing with daily administration and having no effect or decreasing with weekly administration [25]. In this study, TPTD was administered to rats at a frequency of one dose per week on a human equivalent dose basis [6]; the cortical bone porosity decreased, and the cortical bone width increased, as in previous reports [26]. The results of bone histomorphometry in this study correctly reflect the effects of TPTD on the cortical bone. 
Other methods for assessing bone formation include bone formation markers by blood sampling and bone histomorphometry by biopsy. Bone formation markers reflect the metabolism of the whole bone; therefore, sitespecific evaluation was not possible. Furthermore, it is difficult to accurately evaluate the values due to the influence of renal function [27]. Although bone histomorphometry by biopsy can accurately evaluate bone formation, it is necessary to extract the region to be evaluated, which makes it highly invasive. Furthermore, the sample obtained by extraction was also small, and the amount of information was lacking [28]. SWIFT is a non-invasive method that could detect signals reflecting bone formation in a site- and time-specific manner, and it may be used to assess bone formation as an alternative method to bone formation markers or bone histomorphometry by biopsy.

This study had two limitations. First, slight increases in the bone formation rate may not have been detectable as differences in SWIFT signals. Because the rate itself was slow, the proportion of the new bone areas in the cortical bone was small; thus, we believe that the difference in the SWIFT signal could not be detected. We recommend verifying the extent of the bone formation rate that can be detected as a signal in the future. Second, bone formation markers were not measured. Bone formation markers are commonly used to assess bone formation and should be measured to compare the results of SWIFT with those of conventional test. However, we believe that the measurement by bone histomorphometry was sufficient to evaluate the bone formation rate.

\section{Conclusions}

We found that SWIFT could detect increased signals of bound water, reflecting the effect of TPTD on the cortical bone. The signal detected by SWIFT reflects a marked increase in the cortical bone formation rate. We believe that the use of SWIFT to assess bone formation in the cortical bone might help determine the therapeutic effect of TPTD on osteoporosis.

\section{Abbreviations \\ BMD: Bone mineral density; TPTD: Teriparatide; DXA: Dual-energy X-ray absorptiometry; SWIFT: Sweep imaging with Fourier transform; MRI: Magnetic resonance imaging; SNR: Signal-to-noise ratio; ROls: Regions of interest; FA: Flip angle; TR: Repetition time; FOV: Field of view; PDWI: Proton density-weighted image; $\mu \mathrm{CT}$ : Microcomputed tomography; $\mathrm{VBMD}$ : Volumetric bone mineral density; BS: Bone surface; Po Ar: Porosity area: Ct Ar: Cortical bone area; sLs: Single-labeled surface; dLs: Double-labeled surface; MAR: Mineral apposition rate; $\mathrm{BFR/BS}$ : Bone formation rate.}

\section{Acknowledgments}

The authors thank Ms. Akemi Ito, a bone histomorphometric specialist, and the staff at Ito Bone Histomorphometry Institute (Niigata, Japan) for their support.

\section{Authors' contributions}

YS, KI, MK, OO, MM, and HW designed the study. YS mainly performed the procedures. Kl organized this study. Kl, KM, SY, and YS contributed substantially to the writing of the paper. KT supervised the MRI experiment. MT supervised the histological assessments. All authors have read and approved the manuscript.

\section{Funding}

This work was supported by Japan Society for the Promotion of Science (JSPS) KAKENHI [Grant Number 21 K090232].

\section{Availability of data and materials}

The datasets analyzed during the current study are available from the corresponding author on reasonable request.

\section{Declarations}

\section{Ethics approval and consent to participate}

All animal experimental procedures in our study were approved by the Committee for Animal Research, Kyoto Prefectural University of Medicine (number: M2020-285) and carried out in compliance with ARRIVE guidelines. All procedures were performed in accordance with relevant guidelines.

\section{Consent for publication}

Not applicable.

\section{Competing interests}

The authors declare that they have no competing interests.

\section{Author details}

${ }^{1}$ Department of Orthopaedics, Graduate School of Medical Science, Kyoto Prefectural University of Medicine, 465 Kajii-cho, Kawaramachi-Hirokoji, Kamigyo-ku, Kyoto, Japan. ${ }^{2}$ Department of Anatomy and Neurobiology, Graduate School of Medical Science, Kyoto Prefectural University of Medicine, Kyoto, Japan.

Received: 14 Auqust 2021 Accepted: 16 December 2021

Published online: 03 January 2022

\section{References}

1. NIH Consensus Development Panel on Osteoporosis Prevention, Diagnosis, and Therapy. Osteoporosis prevention, diagnosis, and therapy. JAMA. 2001;285(6):785-95

2. Melton LJ 3rd, Khosla S, Atkinson EJ, O'Fallon WM, Riggs BL. Relationship of bone turnover to bone density and fractures. J Bone Miner Res. 1997;12:1083-91.

3. Gambert SR, Schultz BM, Hamdy RC. Osteoporosis clinical features, prevention, and treatment. Endocrinol Metab Clin N Am. 1995;24:317-71.

4. Garnero P, Sornay-Rendu E, Chapuy MC, Delmas PD. Increased bone turnover in late postmenopausal women is a major determinant of osteoporosis. J Bone Miner Res. 1996;11:337-49.

5. Fujita T, Inoue T, Morii H, Morita R, Norimatsu H, Orimo H, et al. Effect of an intermittent weekly dose of human parathyroid hormone (1-34) on osteoporosis: a randomized double-masked prospective study using three dose levels. Osteoporos Int. 1999;9:296-306.

6. Hori M, Uzawa T, Morita K, Noda T, Takahashi H, Inoue J. Effect of human parathyroid hormone (PTH(1-34)) on experimental osteopenia of rats induced by ovariectomy. Bone Miner. 1988;3:193-9.

7. Sugimoto T, Nakamura T, Nakamura Y, Isogai Y, Shiraki M. Profile of changes in bone turnover markers during once-weekly teriparatide administration for 24 weeks in postmenopausal women with osteoporosis. Osteoporos Int. 2014;25:1173-80.

8. Holzer G, von Skrbensky G, Holzer LA, PichI W. Hip fractures and the contribution of cortical versus trabecular bone to femoral neck strength. J Bone Miner Res. 2009;24:468-74.

9. Rockoff SD, Sweet E, Bleustein J. The relative contribution of trabecular and cortical bone to the strength of human lumbar vertebrae. Calcif Tissue Res. 1969;3:163-75. 
10. Idiyatullin D, Corum C, Park JY, Garwood M. Fast and quiet MRI using a swept radiofrequency. J Magn Reson. 2006;181:342-9.

11. Sukenari T, Horii M, Ikoma K, Kido M, Hayashi S, Hara Y, et al. Cortical bone water changes in ovariectomized rats during the early postoperative period: objective evaluation using sweep imaging with Fourier transform. J Magn Reson Imaging. 2015;42:128-35.

12. Minami M, Ikoma K, Horii M, Sukenari T, Onishi O, Fujiwara H, et al. Usefulness of sweep imaging with Fourier transform for evaluation of cortical bone in diabetic rats. J Magn Reson Imaging. 2018;48:389-97.

13. Weiger M, Pruessmann KP, Hennel F. MRI with zero echo time: hard versus sweep pulse excitation. Magn Reson Med. 2011;66:379-89.

14. Kalu DN. The ovariectomized rat model of postmenopausal bone loss. Bone Miner. 1991;15:175-91.

15. Parfitt AM, Drezner MK, Glorieux FH, Kanis JA, Malluche H, Meunier PJ, et al. Bone histomorphometry: standardization of nomenclature, symbols, and units. Report of the ASBMR Histomorphometry nomenclature committee. J Bone Miner Res. 1987;2:595-610.

16. Kanda Y. Investigation of the freely available easy-to-use software "EZR" for medical statistics. Bone Marrow Transplant. 2013;48:452-8.

17. Cullum ID, Ell PJ, Ryder JP. X-ray dual-photon absorptiometry: a new method for the measurement of bone density. Br J Radiol. 1989;62:587-92.

18. Kishi T, Hagino $H$, Kishimoto $H$, Nagashima $H$. Bone responses at various skeletal sites to human parathyroid hormone in ovariectomized rats: effects of long-term administration, withdrawal, and readministration. Bone. 1998;22:515-22.

19. Müller R, Van Campenhout H, Van Damme B, Van Der Perre G, Dequeker J, Hildebrand T, et al. Morphometric analysis of human bone biopsies: a quantitative structural comparison of histological sections and microcomputed tomography. Bone. 1998;23:59-66.

20. Ito M, Oishi R, Fukunaga M, Sone T, Sugimoto T, Shiraki M, et al. The effects of once-weekly teriparatide on hip structure and biomechanical properties assessed by CT. Osteoporos Int. 2014;25:1163-72

21. Horch RA, Nyman JS, Gochberg DF, Dortch RD, Does MD. Characterization of $1 \mathrm{H}$ NMR signal in human cortical bone for magnetic resonance imaging. Magn Reson Med. 2010;64:680-7.

22. Du J, Bydder GM. Qualitative and quantitative ultrashort-TE MRI of cortical bone. NMR Biomed. 2013;26:489-506.

23. Wronski TJ, Yen CF. Anabolic effects of parathyroid hormone on cortical bone in ovariectomized rats. Bone. 1994;15:51-8.

24. Robinson RA. An electron-microscopic study of the crystalline inorganic component of bone and its relationship to the organic matrix. J Bone Joint Surg Am. 1952;34-A(2):389-435.

25. Zebaze R, Takao-Kawabata R, Peng Y. Increased cortical porosity is associated with daily, not weekly, administration of equivalent doses of teriparatide. Bone. 2017;99:80-4.

26. Hodsman AB, Kisiel M, Adachi JD. Histomorphometric evidence for increased bone turnover without change in cortical thickness or porosity after 2 years of cyclical hPTH(1-34) therapy in women with severe osteoporosis. Bone. 2000;27:311-8.

27. Chavassieux P, Portero-Muzy N, Roux JP, Garnero P, Chapurlat R. Are biochemical markers of bone turnover representative of bone histomorphometry in 370 postmenopausal women? J Clin Endocrinol Metab. 2015;100:4662-8.

28. Al Badr W, Martin KJ. Role of bone biopsy in renal osteodystrophy. Saudi J Kidney Dis Transpl. 2009;20:12-9.

\section{Publisher's Note}

Springer Nature remains neutral with regard to jurisdictional claims in published maps and institutional affiliations.
Ready to submit your research? Choose BMC and benefit from:

- fast, convenient online submission

- thorough peer review by experienced researchers in your field

- rapid publication on acceptance

- support for research data, including large and complex data types

- gold Open Access which fosters wider collaboration and increased citations

- maximum visibility for your research: over $100 \mathrm{M}$ website views per year

At $\mathrm{BMC}$, research is always in progress.

Learn more biomedcentral.com/submissions 